\title{
A Proposed Multi Images Visible Watermarking Technique
}

\author{
Ruba G. Al-Zamil \\ Department of Computer Information Systems \\ The Hashemite University \\ Zarqa, Jordan
}

\author{
Safa'a N. Al-Haj Saleh \\ Department of Software engineering \\ The Hashemite University \\ Zarqa, Jordan
}

\begin{abstract}
Visible watermarking techniques are proposed to secure digital data against unauthorized attacks. These techniques protect data from illegal access and use. In this work, a multi visible watermarking technique that allows embedding different types of markers into different types of background images has been proposed It also allows adding multiple markers on the same background image with different sizes, positions and opacity levels without any interference. The proposed technique improves the flexibility issues of visible watermarking and helps in increasing the security levels. A visible watermarking system is designed to implement the proposed technique. The system facilitates single and multiple watermarking as illustrated in the proposed technique. Experimental results indicate that the proposed technique applies visible watermarking successfully.
\end{abstract}

Keywords-Visible watermarking; multi-image watermarking; marker image; background image; image channels; opacity; Matlab

\section{INTRODUCTION}

Digital data distribution is widely spread due to the rapid growth of networks and internet communications in the recent years. The trend of moving from traditional libraries to digital libraries is growing day after day. The availability of digital data- such as images, sounds, videos, and e-books- may lead to slick illegal duplication or distribution; so it should be protected by contractual and intellectual property rights.

To regulate copyright issues and to prevent unauthorized use, the concept of digital watermarking has evolved over the past few years. It basically depends on embedding a digital signal with information that cannot be easily removed [12]. Generally, digital watermarking is the process of hiding any digital message -usually called marker or watermark- into a digital media. The embedded message may be either visible or invisible. When dealing with images, it is common to embed digital marker image or text into digital host image such as textual data about the author, or copyright image. The main goal of image watermarking is to protect confidentiality, integrity, availability and authenticity of images [15] taking into consideration that watermarking process should not affect the quality of original and watermarked image.

According to human perception, image watermarking techniques can be divided into three types [16]: Visible watermark, invisible watermark, and dual watermark. Visible watermarking is a semi-transparent text or image (i.e. the marker) that is overlaid on the original image where the watermark is visible to the viewer. The used watermark often contains name or logo of company or copyright information that identifies the owner of the original image. When adding a visible watermark, it is important to take into consideration that the marker should be difficult to remove; i.e. removing the watermark should be more costly than purchasing the original image from the owner. In invisible watermarking the watermark is added to the original image in a way that no modifications are noticed; i.e. the watermark is hidden in the image and can be retrieved by some mathematical calculations to prove ownership. Dual watermarking is the combination of visible and invisible watermark where invisible watermark is used as a backup for the visible watermark.

Some desirable properties should be applied on watermarking process to get the intended goals [8]. These properties include effectiveness (i.e. the watermark should be detective), quality of watermarked image, watermark size, and watermark robustness [15] against attacks.

Watermarking has many applications where the issues of security and ownership preservation are vital. It is applied in owner identification, copy and usage control [17] [4], broadcast monitoring, medical applications, fingerprinting, and military applications.

In this work, a visible watermarking technique for embedding different types of multi watermarks into background images is proposed.

The paper is organized as follows: Section 2 presents related work and previous proposed techniques. The proposed multi visible watermarking technique is discussed in section 3 . Visible watermarking system that implements the proposed technique is presented in section 4 . In section 5 the results are reported and appropriate discussion is made. Finally, the paper is closed with a conclusion in section 6 .

\section{RELATED WORK}

Many techniques and methodologies were proposed for visible and invisible watermarking. In this section, we outline some visible watermarking techniques as mentioned in the literature. Mohanty et al. [14] presented a visible watermarking scheme that is applied to the original image in the DCT domain based on a developed mathematical model. They have also proposed a modification to increase the robustness of the watermark. Tamilselvi et al. [6] proposed a methodology based on DCT modification of original image with respect to watermark image. The watermarking is done in frequency domain using compound mapping function. The 
results showed that the proposed watermarking process is secure against possible attacks. Parvathavarthini et al. [13] proposed a watermarking scheme using Hadamard transform based on a calculated scaling factor of the image. Hadamard transform was used due to its robustness against attacks. The value of scaling factor was controlled by a control parameter. The proposed scheme proved its efficiency by experimental results and performance analysis. Jose et al. [3] suggested a new approach for lossless visible watermarking using one to one compound mapping. Two types of applications are were proposed, opaque monochrome watermarks and nonuniformly translucent full color watermarks.

A new method was proposed by Bhaisare et al. [9] for visible watermarking for lossless image recovery capability based on one-to-one compound mappings. The methodology was designed to embed different types of visible watermarks into images. Majeed et al. [10] presented a large scale integration (VLSI) architecture for implementing two visible digital image watermarking schemes. The authors designed a watermarking chip that can be integrated within a digital camera. The integrated chip can have two different types of watermarking capabilities in spatial domain. Kumar et al. [7] presented a method based on the use of deterministic one-toone compound mappings of image pixel values to watermark visible watermarks on original images. Park and Kim [5] proposed a watermarking technique using digital seal image (i.e. a binary image) as a marker. The authors showed how to construct a verifiable seal image and how to apply it to the original image. Biswas et al. [2] suggested a methodology for adding watermarks to medical images in Region of NonInterest (RONI) to guarantee correct diagnosis. The selection of RONI was based on Fuzzy C-Means segmentation and Harris corner detection. Watermark embedding was based on alpha blending technique. Chen et al. [1] presented a wavelet based visible watermarking scheme that partitioned the original image to four similar images. The partitioned images were classified into two sets: fixed set (FS) and water marked set (WS). Wavelet transform was applied on the partitioned images for embedding watermark image.

Most of the presented related works do not present embedding different types of markers into different types of background images, and adding multi markers on the same background image.

\section{ThE PROPOSED MUlTi VisiBLE WATERMARKING METHODOLOGY}

The proposed technique allows adding multiple markers on the same background image by selecting different positions for hiding the markers and preventing any interference between them. It allows hiding markers with different transparency degrees using different opacity levels. Repeating any marker with different size, position, and opacity is also allowed.

There is no restriction to use a specific type of images for marker and background images. Different types of images can be used (i.e. binary, gray, and RGB). Any combination of marker and background images is valid; for example RGB background may contain binary, gray, or RGB markers. On the other side, gray background may contain binary, gray, or RGB markers and so on.

Suppose that we tend to hide image 1(marker image) in image 2 (background image). The proposed technique is based on the following:

1) Store the state of images. The stored state indicates images types (i.e. binary, grayscale, or RGB).

2) Resize watermark image to suitable size smaller than the original image size.

3) The position where the marker image will be inserted is specified. This position represents the center point of the marker image. According to the center point, the start point (i.e. the top-left corner) and the end point (i.e. the bottom-right corner) are calculated. This is to check that the marker will not exceed the boundaries of the background image.

If the start or the end points are outside the background image, the insertion position will be modified to be within the boundaries of the background image.

4) Store number of channels for each image depending on its type; i.e. three channels for RGB image, and one channel for gray or binary image. This is because embedding marker image into background image is based on merging the corresponding channels.

5) Marker image is added with a specific level of transparency (i.e. opacity). Opacity values can be between $0-1$. Transparency is usually not considered to be a blending mode. Transparency is just a combination of the multiply and addition blending modes; it takes a percentage of the foreground (i.e. marker image) and adds it to the complementary percentage of the background image. Thus, if we want the value of the foreground to be $75 \%$ opaque, multiply the foreground by 0.75 and the background by (10.75 ) and add the two values.

The proposed technique applies the following transparency equation (1) [11]:

$$
K=n * A+(1-n) * B
$$

where $\mathrm{K}$ refers to the output image, $\mathrm{n}$ refers to opacity value, $\mathrm{A}$ refers to the marker image and $\mathrm{B}$ refers to the background image.

6) After applying this equation the final result (output image) will be converted to RGB image. This is to view and preserve all colors in the combined images (i.e. marker and background images). For example, suppose we have binary marker embedded into RGB image, if final image is stored as binary or gray, colors in RGB are not preserved. On the other side, suppose we have binary marker embedded into gray image, there is no problem to store final image as RGB as it will preserve colors in binary and gray images.

7) Finally, The width, the height, the size and the position of the marker image are stored. This helps in adding new markers without any conflict (i.e. markers interference). This will be explained in details in the next section. 


\section{VISIBLE WATERMARKING SYSTEM}

A visible watermarking system is designed using Matlab 7.8.0 (R2009a) to implement the proposed technique. The system facilitates loading marker and background images. It allows the user to choose marker position by specifying $\mathrm{X}$ and $Y$ coordinates. The user can also determine the opacity and the size of the added marker image. Fig 1 shows the main screen of visible watermarking system.

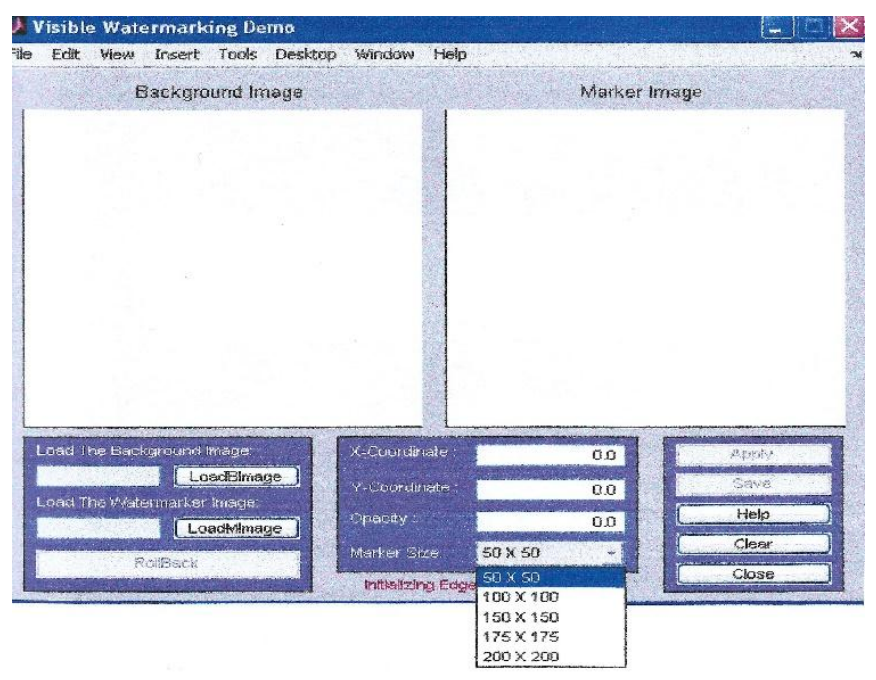

Fig. 1. Visible watermarking system

The system provides the following functionalities:

- Background and marker images selection and loading.

- Marker's position selection (i.e. center point). The user can determine the position using mouse. By this, the values of $\mathrm{X}$ and $\mathrm{Y}$ coordinates will be determined automatically. Coordinates values can be also entered manually.

- Opacity value selection within the range of (0-1). The value of 0 , means that the marker will not appear in the background image at all (i.e. invisible watermarking). The value of 1 means that the marker will replace the selected part of the background image. The values between 0 and 1 will view the marker in a transparent mode.

- Marker size determination. User can choose one of the following sizes: (50X50, 100X100, 150X150, 175X175, and 200X200). If the selected marker size is greater than the background image, the user will be notified to choose another smaller size.

The user should apply all the specified options to get the final result (i.e. the watermarked image). When applying watermarking process, it is common that the user needs to rollback and go back to the previous state. To make this applicable, the system saves all watermarking steps in a stack. When the user clicks the rollback button, the system will remove the latest watermarking step. If the user chooses to roll back again, the watermarking step before the latest one will be removed and so on. When the user chooses another background image the stack will be cleared.

The user can add another marker(s) to the watermarked image. The system prevents markers overlapping by storing the position and the size for each added marker. When the user adds a new marker, the system compares the current position and size with all markers added before, if any interference occurs the user will be notified.

Markers overlapping can occur in two cases:

1) The user selects the same $X$ and $Y$ coordinates for an added marker on the background image.

2) The user selects different coordinates but unexpected part of the new marker overlaps an added marker. In this case, the user needs to make sure that the new selected position is away enough from other marker images around the selected position for the added marker.

\section{RESULTS AND DISCUSSION}

This section presents the experimental results of the proposed technique. The functionality of the implemented system is tested by performing watermarking on number of test images. Marker and background test images are of various types and sizes.

The proposed technique applies visible watermarking successfully on test images.

Fig 2 shows sample of some results of embedding different types of markers into background images where (a) shows RGB marker embedded into RGB background image, (b) shows RGB marker embedded into binary background, (c) shows grayscale marker embedded into grayscale image, and (d) shows binary marker embedded into grayscale image.

The proposed technique also allows embedding multiple markers into the same background image with no interference. Fig 3 shows an example. Also embedding repeated marker with different size and positions is also allowed. Fig 4 illustrates an example using camera man as a marker.


Fig. 2. Sample of some results of the proposed technique 


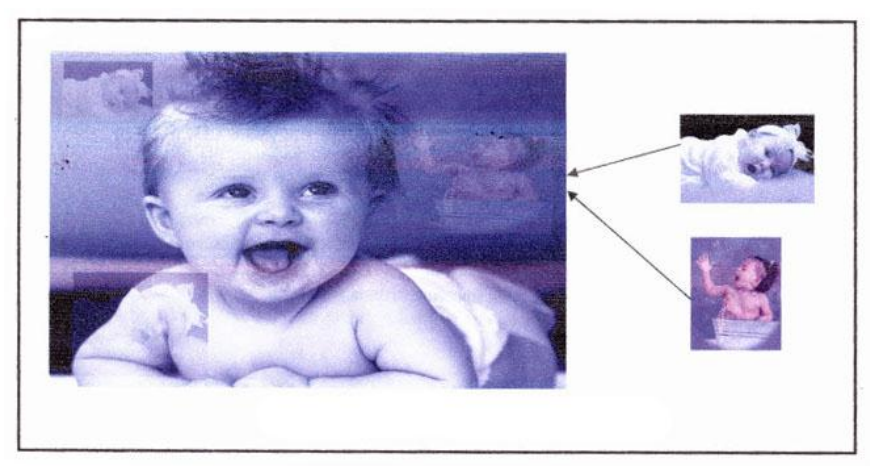

Fig. 3. Embedding multiple markers into the same background image

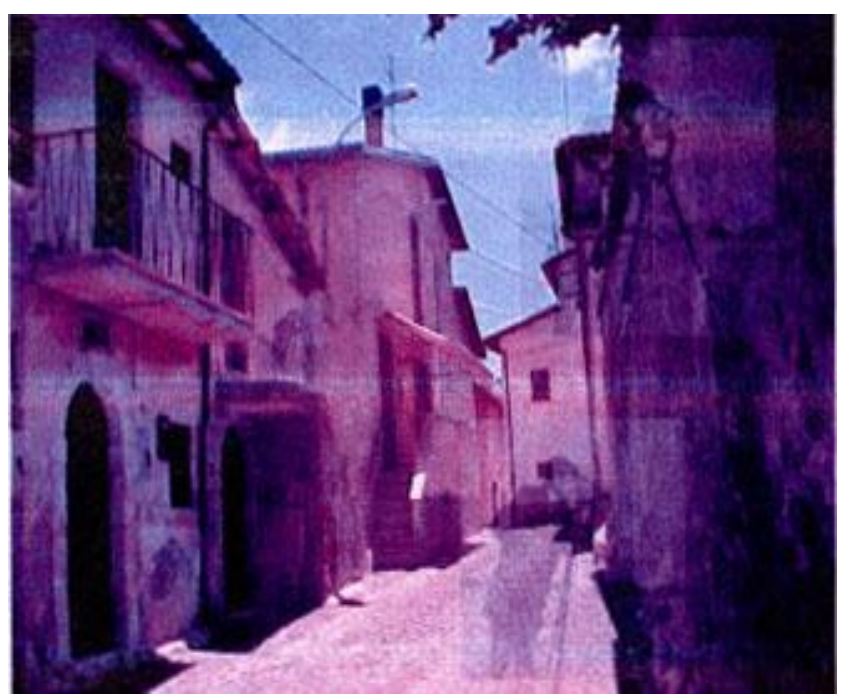

Fig. 4. An example of adding repeated marker with different size and positions

\section{CONCLUSION}

A multi visible watermarking technique has been proposed. The proposed technique has presented adding different types of images into different types of background images. Embedding multiple markers on the same background image with no interference has been also proposed. Multi visible watermarking technique is based on storing the state of images (images types), resizing marker image to suitable size smaller than the original image size, determining insertion position of marker image, embedding marker image with background image based on number of channels using transparency equation, and storing width, height, and position of the marker image to prevent markers interference in the case of adding many markers. A visible system has been also designed to implement the proposed technique. The system provides many functionalities to allow single and multiple watermarking with different sizes, positions, and opacity levels.

For future work, we aim to embed animated watermarks. We also tend to expand the functionalities of the designed system to allow the user to enter the desired watermark as a text and embed this text into background image

\section{REFERENCES}

[1] C. Chen , and H. Tsai, "Wavelet-Based Reversible and Visible Image Watermarking Scheme", Springer-Verlag Berlin Heidelberg ,2011.

[2] D. Biswas, P. Das, P. Maji, N. Dey and S. Chaudhuri, "Visible Watermarking Within the Region of Non-Interest of Medical Images based on Fuzzy C-Means And Harris Corner Detection", Third International Conference on Computer Science, Engineering and Applications (ICCSEA 2013), Delhi, India, 24-26 May 2013.

[3] D. Jose, R. Karuppathal, and A. Kumar, "Copyright Protection using Digital Watermarking", National Conference on Advances in Computer Science and Applications with International Journal of Computer Applications (NCACSA), 2012.

[4] G. Kaur and K. Kaur, "Digital Watermarking and Other Data Hiding Techniques", International Journal of Innovative Technology and Exploring Engineering (IJITEE) ISSN: 2278-3075, Volume-2, Issue-5, April 2013.

[5] H. Park and K.Kim, "Visible Watermarking using Verifiable Digital Seal Image", Symposium on Cryptography and Information Security, pp. 103- 108, Japan, 2001.

[6] J. Tamilselvi and S. Janakiraman, "A Visible Watermarking Scheme for DigitalImages in Frequency Domain", International Journal of Advanced Networking and Applications (IJANA), Vol. 4 Issue 3 pp. $1635-1639,2012$.

[7] K.Madhu Kumar, M. Katta Swamy and B. Reddy, "Lossless Visible Watermarking Using Compound Mapping", International Journal of Engineering Research and Development, Volume 4, Issue 9, PP. 2735,Nov. 2012.

[8] L. Saini and V. Shrivastava, "A Survey of Digital Watermarking Techniques and its Applications", International Journal of Computer Science Trends and Technology (IJCST), Volume 2 Issue 3, May-Jun 2014

[9] M. Bhaisare and V. Raut, "Generic Lossless Visible Watermarking: A Review" ,International Journal of Innovative Research in Computer and Communication Engineering, Vol. 3, Issue 1, January 2015.

[10] M. Majeed , S.C.Ramesh and R.Anuja, "Implementation of a Visible Watermarking in a Secure Still Digital Camera Using VLSI Design", International Symposium on Computing, Communication, and Control (ISCCC), 2009.

[11] M. Topkara, A. Kamra, and M. J. Atallah, "ViWiD: Visible Watermarking Based Defense against Phishing", Lecture Notes in Computer Science, Vol.3710, pp.470-483., 2005,

[12] P. Bidla, S. Gengaje, and R. Shelke, "Visible Image Watermarking Based On Texture and Luminance Blocks In DCT Domain - A Review", International Journal of Emerging Technology and Advanced Engineering, Volume 2, Issue 4, April 2012.

[13] S. Parvathavarthini and R. Shanthakumari, "An Adaptive Watetmarking Process In Hadamard Transform", International Journal of Advanced Information Technology (IJAIT),Vol. 4, No. 2, April 2014.

[14] S. P. Mohanty, K. R. Ramakrishnan and M. S. Kankanhalli, "A DCT domain visible watermarking technique for images", Proc. IEEE Int. Conf. Multimedia Expo., vol. 2 , pp.1029 -1032, 2000.

[15] S. Priya, B. Santhi and P. Swaminathan, "Image Watermarking Techniques - A Review", Research Journal of Applied Sciences, Engineering and Technology, July 2012.

[16] V. Singh, "Digital Watermarking: A Tutorial", Multidisciplinary Journals in Science and Technology, Journal of Selected Areas in Telecommunications (JSAT), Jan. 2011.

[17] Y. Perwej, F. Parwej and A. Perwej, "An Adaptive Watermarking Technique for the copyright of digital images and Digital Image Protection", The International Journal of Multimedia \& Its Applications (IJMA) Vol.4, No.2, April 2012. 